\title{
TRANSPARENCIA PRESUPUESTAL DE LAS ENTIDADES SUBNACIONALES EN COLOMBIA
}

\section{BUDGET TRANSPARENCE OF SUB-NATIONAL ENTITIES IN COLOMBIA}

\author{
Jairo Alonso Bautista' \\ Didier Sepúlveda Reyes² \\ Adriana Marcela Mantilla Salamanca ${ }^{3}$
}

\section{RESUMEN}

El presente avance investigativo tiene como propósito realizar un análisis sobre los niveles de Transparencia Presupuestal (TP) de la gestión fiscal y financiera de los departamentos en Colombia, mediante la aplicación de la metodología, empleada por el Índice de Transparencia Presupuestal, desarrollada por el International Budget Partnsership.

Estudios anteriores han señalado los bajos niveles de participación ciudadana en la construcción, seguimiento

1 Contador de la Universidad Nacional de Colombia, magíster en Administración Pública de la ESAP. Docente investigador de la Universidad Santo Tomás. Miembro del grupo de investigación: Contaduría: Información, Control e Impacto Social, adscrito a la Universidad Santo Tomás.

Correo electrónico: abautistaj@unal.edu.co Orcid: https://orcid.org/0000-0003-1344-1692

2 Administrador público de la Escuela Superior de Administración pública (ESAP), investigador junior del grupo Ciudadanía y Finanzas Públicas, adscrito a la ESAP. Estudiante de la Maestría en Planeación para el Desarrollo Universidad Santo Tomás (USTA).

Correo electrónico: didier.sepulveda155@esap.gov.co Orcid: https://orcid.org/0000-0002-9766-2662

3 Administradora pública de la Escuela Superior de Administración pública (ESAP), investigadora del grupo Ciudadanía y Finanzas Públicas, adscrito a la ESAP. Especialista en Gerencia Social de la ESAP.

Correo electrónico: adriana.mantilla206@esap.gov.co

Orcid: https://orcid.org/0000-0003-1004-3859

Código JEL: B41, H61, H83.

Fecha de recepción: 21/08/2018

Fecha de aprobación: 22/09/2018

DOI: https://doi.org/10.18601/16577175.n22.03 
y vigilancia del presupuesto público, particularmente en las regiones menos desarrolladas del país, en las que las instituciones democráticas aún se muestran débiles e incipientes. Estos bajos niveles de participación se explican también por los bajos niveles de transparencia de la información presupuestal, la cual dificulta el ejercicio ciudadano del control y la participación ciudadana.

En este sentido, la transparencia debe garantizar no solo un deber del Estado, sino que también un derecho ciudadano con el fin de permitir: i. Controlar la ejecución eficaz y eficiente del presupuesto, ii. Hacer incidencia para modificarlo y iii. Evaluar los impactos del gasto en aspectos como mejora de la calidad de vida y disponibilidad de mayores y mejores bienes públicos (International Budget Partnership, 2015).

La propuesta fundamenta la construcción de los elementos analíticos para evaluar la transparencia y permitir la creación de un estudio de largo plazo que permita verificar la evolución en el tiempo de los niveles de transparencia del presupuesto de los departamentos y sirva de guía para el fortalecimiento de prácticas de transparencia presupuestal.

Palabras clave: transparencia, información fiscal, disponibilidad, comprensibilidad, oportunidad, presupuesto, departamentos.

\section{ABSTRACT}

This research progress seeks to analyze the Budgetary Transparency of fiscal and financial management through the application of the Budget Transparency Index, developed by the International Budget Partnership in the administrations of the 32 departments in Colombia, and evaluating the quality of the budget information prepared and disseminated by departmental administrations.

The foundations of the study are determined by the low levels of citizen participation in the construction, monitoring and surveillance of the public budget, particularly in the less developed regions of the country, where democratic institutions are still weak and incipient.

Under these precepts, transparency must guarantee not only a duty of the State, but also a citizen's right in order to allow I. Control the effective and efficient execution of the budget, II. Make an impact to modify it and III. Evaluate the impacts of spending on aspects such as improving the quality of life and availability of greater and better public goods (International Budget Partnership, 2015).

Keywords: tax information, availability, comprehensibility, opportunity, budget, departments.

\section{INTRODUCCIÓN}

En los últimos treinta años, en el contexto internacional y regional, las demandas por mayores niveles de transparencia de los gobiernos se han convertido en el origen de numerosos esfuerzos institucionales por proporcionarle a los ciudadanos altos niveles de información y acceso a las decisiones que toman los gobernantes, en especial, 
aquellas decisiones que tienen relación con el uso de los recursos del presupuesto público.

Las exigencias por mayor transparencia, coinciden con un aumento de la desconfianza de los ciudadanos en las acciones y decisiones de sus gobernantes, lo que en Colombia se convierte - gracias a fenómenos como la corrupción - en un problema que amenaza no solo con minar aún más la credibilidad de los gobiernos, sino el reforzamiento de actitudes negativas hacia las instituciones estatales, la desintegración social y la prevalencia del conflicto social.

En este marco, la Transparencia Presupuestal se ha convertido en un requisito esencial para evaluar el desempeño de la gestión de los gobernantes: conocer, comprender, disponer de la información libremente, realizar seguimiento e influir en las decisiones presupuestales se convierten en sinónimos de democratización del gobierno y de madurez de las instituciones sociales y política de un país.

Si bien, para las finanzas y el proceso presupuestal del nivel central de gobier- no se han desarrollado diversos instrumentos de medición de sus niveles de transparencia presupuestal en la última década ${ }^{4}$, para los niveles subnacionales no se ha avanzado en una medición y evaluación de la transparencia presupuestal a diferencia de países líderes en este proceso como México y Brasil. En Colombia es muy escaso el conocimiento del desempeño de las finanzas territoriales desde una perspectiva de transparencia ${ }^{5}$.

Conscientes de este contexto, y de las deficiencias en la medición y evaluación de la transparencia en los niveles subnacionales en Colombia, el grupo de investigación Ciudadanía y Finanzas Públicas de la Escuela Superior de Administración Pública ha propuesto la creación del Índice de Transparencia Presupuestal de los departamentos en Colombia, siguiendo estándares desarrollados por reconocidas agencias e instituciones globales -Fondo Monetario Internacional, Organización para la Cooperación y el Desarrollo Económicos (OCDE) y el Open Budget Partnership - como herramienta para identificar, evaluar y promover mayores niveles de transparencia de los

4 En el 2011, el PEFA (Public Expenditure and Financial Accountability) emitió un reporte sobre transparencia presupuestal en el que se describieron los resultados para Colombia del modelo. En el 2011, 2013, 2015, 2017, el Open Budget Survey realizó evaluaciones del nivel de transparencia de las finanzas del nivel central del Gobierno y realizó un ranking con un amplio número de países del mundo.

5 Es claro que el nivel central del gobierno produce información sistemática de aspectos del desempeño fiscal de gobernaciones y municipios, sin embargo, su interés es más de control de los niveles de endeudamiento que un análisis sistemático de la producción, circulación, uso y comprensión de las cifras fiscales por parte de la ciudadanía. Adicional a ello, instituciones como Transparencia por Colombia han realizado indicadores de transparencia territorial, pero su enfoque es más sobre información y procesos genéricos que sobre información presupuestal en específico, aunque son una muy buena aproximación a la estructura del presente estudio. 
presupuestos públicos de los departamentos, así como para el desarrollo de políticas de gestión de la información que permitan el fortalecimiento de los canales de participación y control ciudadano, además del mejoramiento de los marcos de decisiones de los gobernantes sobre las finanzas de sus territorios.

\section{MATERIALES Y MÉTODOS}

Los estudios sobre transparencia presupuestal en países latinoamericanos tienen antecedentes muy escasos y especialmente en la evaluación o análisis de transparencia de los entes subnacionales. En los últimos años la producción de publicaciones de la línea de las finanzas públicas ha presentado temas presupuestarios en relación con su coordinación, proceso, normatividad, evaluación y se ha orientado a resultados de políticas fiscales.

La transparencia presupuestal debe ser vista como la divulgación completa de toda la información fiscal pertinente de manera oportuna y sistemática (Organización para la Cooperación y el Desarrollo Económicos [OCDE], 2001). Existen factores más profundos que pueden afectar los niveles de apertura gubernamental y, posteriormente, la transparencia presupuestaria. La transparencia presupuestaria se considera importante para fomentar un debate abierto sobre si los recursos públicos se utilizan para satisfacer las necesidades de la población. Este tipo de debate democrático no es característico de la cultura política en muchos países pobres (Renzio, 2011).
Por otra parte, la transparencia se ve como un medio para mejorar la gobernanza económica por medio de una combinación de mecanismos, fortalecimiento, rendición de cuentas y legitimidad, así como facilitar coherencia política y previsibilidad y promoviendo el mejor funcionamiento del gobierno (Heald, 2003).

De esta manera, la transparencia presupuestal pretende generar al mercado eficiencias, reducir los costos de endeudamiento, facilitar el intercambio de información y el poder de suavizar la inversión extranjera directa y otros flujos de capital (Bessire, 2005; Glennerster y Shin, 2003). Los presupuestos transparentes, en particular, fomentan la facilidad de acceso a una actividad fiscal consolidada, contiene auditoría, procedimientos, se carece de cuentas especiales y se difunden datos (Poterba y Von Hagen, 1999). Otro factor importante de la transparencia del presupuesto es que ayuda a los interesados a evaluar las condiciones fiscales de una organización, comprender sus costos y los beneficios de sus servicios.

En el campo de la transparencia presupuestal, la participación pública se manifiesta como una solución y como un apoyo hacia la toma de decisiones de las políticas fiscales generando así un mayor grado de responsabilidad por parte de la sociedad y los entes reguladores, estas decisiones deben tomarse abiertamente involucrando a todos los actores que hagan parte de los procesos de formulación, evaluación, implementación, control y seguimiento de dichas políticas (Ríos y Benito, 2016). 
No obstante, para el cumplimiento de estas políticas debe tenerse planeado un presupuesto en el que se reflejen los recursos que se destinarán a planes financieros y misionales.

La transparencia es un calificador que permea todo y cualquier acto, comportamiento, procedimiento, decisión o medida en el cotidiano de la maquinaria pública y de la relación gobierno-sociedad. La Transparencia de las Cuentas Públicas (TCP) busca impedir la práctica de la corrupción en la gestión pública a través de la influencia del control social, es decir, el control ejercido por la sociedad sobre las acciones del Estado. De esta forma, TCP puede ser considerada un medio de información social que debe ser adecuado para la comprensión del público objetivo. En este sentido, se trazan reflexiones sobre la necesidad de actuación de la sociedad, lo que puede fortalecer la contabilidad de los gestores públicos ante los ciudadanos (Rausch y Soares, 2010).

Paprocki (2017), verifica el nivel de transparencia de las informaciones sobre la gestión pública municipal y cuáles características e indicadores socioeconómicos de los municipios influencian el nivel de transparencia observado. El autor destaca la existencia de asociación entre las condiciones socioeconómicas de los municipios y los niveles de transparencia en la divulgación de informaciones sobre la gestión pública

Por otra parte, la transparencia en las finanzas públicas y en la gestión fiscal implica la sistemática, completa y oportuna puesta a disposición del público en general (divulgación) de las informaciones económico-financieras gubernamentales, relacionándolas a las funciones, objetivos, intenciones y acciones de los responsables, las decisiones que involucran recursos públicos en las fases de planificación, ejecución, control y evaluación en las diversas estructuras de gobierno (Pires, 2014).

Entre otros aspectos la transparencia puede contener secciones como (Álvarez, 2006):

- Presupuestos por programas

- Identificación de los montos involucrados.

- Identificación del propósito del gasto.

- Identificación del responsable del gasto.

- Mirada estratégica a través de presupuestos plurianuales

- Participación y rendición de cuentas

- Análisis y discusión parlamentaria.

- Evaluación de los resultados.

- Presupuestos participativos.

Igualmente, debe considerarse que el presupuesto está compuesto por procesos y cada parte de este proceso es transparente cuando permite a todos los agentes con interés en el proceso (gobierno, instituciones de control interno y externo, mercados y ciudadanos) conocer la verdadera situación de las finanzas públicas y verificar el cumplimiento de los objetivos presupuestarios (Betriu, 2003).

Desde otra perspectiva, la Transparencia Presupuestal puede ser entendida 
como política pública que está asociada a la profundización democrática, a la buena gobernanza y a la prevención y combate a la corrupción. Por tanto, tales políticas pueden ser consideradas eficientes si contribuyen a profundizar los niveles de democracia de los países, a garantizar o mejorar la gestión de las políticas públicas y a prevenir y combatir la corrupción (Moreira y Claussen, 2011). Cabe mencionar, que la verdadera Transparencia Presupuestal puede permitir al ciudadano analizar las escogencias realizadas respecto a la asignación de los recursos públicos, por ejemplo, sobre cuánto el Estado invierte en la obra de una escuela, en la contratación de un determinado servicio o incluso en la compra de garrafas de agua mineral. No solo por exigir el desarrollo de un sistema de finanzas y control que dé soporte a la gestión de las finanzas públicas, sino también por exigir que los datos disponibles por todos los poderes y entes presenten un nivel de detalle mínimo y estandarizado.

Otra cuestión fundamental que se relaciona con la postura de la Transparencia Presupuestal como política pública es la de "gobierno abierto", que no se limita a la transparencia presupuestaria. Otros autores como Gómez y Montesinos (2014) destacan el término de gobierno abierto, que también lo llama gobierno electrónico o e-government, este emerge y se consolida como un proyecto que impulsa la utilización del internet al servicio de un mejor gobierno. El gobierno electrónico pretende impactar en la transparencia, puesto que supone mejoras en la información.
De la misma manera, los diversos sectores del gobierno sean aptos y estén conscientes de la necesidad de que el ciudadano tenga pleno acceso a la información relacionada con el presupuesto. Así, la traducción del lenguaje técnico a un lenguaje comprensible, la divulgación de datos que permitan análisis comparativos y exámenes agregados, la procura del acceso público a los documentos oficiales y el propio despertar y aprendizaje de los ciudadanos y otros tipos de usuarios de datos e información pública, tales como las empresas, son ejemplos de los obstáculos que requieren ser permanentemente superados (Moreira y Claussen, 2011).

Por otra parte, el poder acceder a información presupuestaria es fundamental para la transparencia del sector público y así aumentar su efectividad y contabilidad. Recientemente, se ha iniciado un movimiento para que los datos sean disponibles libremente, en tiempo real y para cualquier ciudadano. Se trata de un proceso nuevo, aún en construcción, y con características que varían de acuerdo con el país en el que ocurre. El usuario también es responsable de la comprensión de la información, debiendo, si es necesario, estar dispuesto a estudiar el tema. Sin embargo, el responsable primario de la comprensión de la información es la entidad que las divulga.

En este sentido, se identifican tres patrones de divulgación que generalmente se proponen: divulgación adecuada, justa y completa (Hendriksen y Van Breda (1999), citado por Platt, Cruz, Ensslin y Ensslin, 2007). 
En Brasil, la disponibilidad de datos referentes a la gestión pública se intensificó a partir de la aprobación de la Ley de Acceso a la Información (LAI) en el 2011, la cual regula los procedimientos a ser observados por los entes federados con el propósito de garantizar el acceso de la información (Beghin y Zighoni, 2014). Más que garantizar el cumplimiento de las normas legales, las iniciativas de transparencia en la administración pública constituyen una política de gestión responsable que beneficia la práctica de la ciudadanía por parte de la población. Por tanto, la comprensión de los datos y la información por parte de los usuarios es una condición indispensable (Platt, Cruz, Ensslin y Ensslin, 2007).

Es necesario agregar que los gobiernos más transparentes no garantizan gobiernos mejores. Igualmente, más información abierta al público no garantiza ciudadanos más informados. Sin embargo, las medidas y las políticas de transparencia, cuando se diseñan para alcanzar objetivos bien definidos pueden crear condiciones para formular políticas más efectivas y gobiernos menos corruptos. Asegurar la efectividad de las medidas o políticas de transparencia exige la definición de un público objetivo y de estrategias para incorporar a la cotidianidad, de los gestores y de los usuarios, la información divulgada. Incluso, los gobiernos tienen el deber de hacer un esfuerzo para aumentar la transparencia presupuestaria, ya que permite que los ciudadanos y los mercados financieros evalúen adecuadamente la posición y el desempeño financiero del gobierno y mejorar el proceso de toma de decisiones de los agentes económicos (Bastida y Bernardino, 2007).

Para el caso español, Ríos, Guillamon, Benito y Bastida (2017) elaboran un estudio sobre la transparencia presupuestal y el desempeño fiscal en el ámbito municipal, se destaca que el nivel de transparencia municipal influye en las desviaciones de los pronósticos presupuestarios en los ingresos tributarios y los gastos corrientes. Por un lado, los municipios menos transparentes sobreestiman sus ingresos, lo que les permite proporcionar más servicios públicos sin un aumento inmediato de los impuestos. Por otro lado, estos gobiernos locales, conscientes de la sobreestimación de sus ingresos, pueden gastar menos de lo presupuestado. Mientras tanto, las municipalidades más transparentes parecen ser más prudentes en sus estimaciones de ingresos, ya que subestiman sus ingresos, lo que significa que pueden gastar más de lo proyectado.

Los resultados también muestran que el comportamiento de los políticos está influenciado por la fase del ciclo electoral en el que se encuentran y los políticos sobreestiman los gastos en el año anterior a las elecciones. La transparencia depende de los incentivos de los directivos y los políticos para divulgar información precisa y completa. Según International Budget Partnership (2015), el Gobierno español no proporciona suficiente información presupuestaria a los ciudadanos. La falta de transparencia puede aumentar la confusión de los votantes y reducir el compromiso de los políticos de ser fis- 
calmente responsables. La confusión de los votantes y la falta de transparencia pueden evitar un control efectivo del presupuesto, particularmente cuando se necesitan ajustes fiscales sustanciales. La falta de control presupuestario puede promover la manipulación del presupuesto y las desviaciones relevantes.

Para el caso de Colombia, los estudios sobre transparencia presupuestal en Colombia tienen antecedentes muy escasos. Pese a que los últimos quince años se han producido publicaciones e informes en materia de finanzas públicas, en relación con el presupuesto público, estos se han delimitado bajo enfoques analíticos de procesos de coordinación presupuestal, institucional y normativa con orientación hacia resultados de políticas fiscales de consistencia con fundamento a los requerimientos financieros de organismos multilaterales.

Las investigaciones adyacentes a la problemática central descrita en la presente investigación son documentos en el contexto latinoamericano como "Transparencia fiscal, rendición de cuentas y riesgo fiscal" desarrollado por el Fondo Monetario Internacional (FMI, 2012), en el que su objetivo central es la medición de una relación positiva entre el grado de transparencia fiscal y las medidas de sustentabilidad fiscal, es decir, la implementación de acciones de transparencia fiscal mediante la construcción normativa para el desempeño fiscal de los países latinoamericanos. Los resultados arrojados para el caso particular de Colombia fue que esencialmente existen considerables brechas e inconsistencias en las normas de trans- parencia fiscal en áreas tales como la cobertura de las instituciones públicas, el tratamiento de activos y pasivos, la presentación de informes sobre transacciones y otros flujos económicos, así como la comparación entre los datos proyectados y reales (FMI, 2012).

En el ámbito local, los análisis sobre la trasparencia presupuestal han sido menos abundantes, dado que los avances sobre la materia se establecen en las dinámicas del presupuesto como proceso político en el que intervienen actores de las instancias del poder ejecutivo y legislativo, y en menor medida la sociedad organizada, mediante mecanismos democráticos con sujeción estricta a las leyes nacionales.

En este aspecto, los estudios abandonan los pilares de la rendición de cuentas, principalmente en hacer público el presupuesto público como principio de la transparencia y se enfilan hacia caracterizaciones de sostenibilidad, adaptabilidad, eficiencia y representatividad del presupuesto público, incidencia normativa que trajo consigo la Constitución Política de Colombia de 1991. Entre los estudios se cuenta el elaborado por el Banco Interamericano de Desarrollo (BID, 2006) y Fedesarrollo: "La economía política del proceso presupuestal en Colombia". Las conclusiones determinan que el proceso presupuestal resulta incluyente y participativo en parte por la observancia inflexible a las reglas fiscales, pese a la falta de adaptabilidad, eficiencia y sostenibilidad (BID, 2006).

Ahora bien, el (International Budget Partnership, 2015) ha indicado que el 
análisis de la transparencia presupuestal implica una valoración sobre los siguientes elementos:

a. ¿A través de qué medios se informa? (medios virtuales, impresos, medios de comunicación masiva).

b. ¿Qué tipo de información se produce para los diferentes públicos?

c. ¿Qué tipo de pedagogía se utiliza para que el público en general comprenda la información presupuestal?

Sin embargo, la Organización para la Cooperación y el Desarrollo Económicos (OECD) (2010) en el documento "Las mejores prácticas para la transparencia presupuestaria" determinó que la transparencia, como una herramienta de apertura con respecto a las intenciones, a la formulación e instrumentación de la política pública como clave del buen gobierno, se expresa en tres dimensiones: los tipos de reportes que se producen, la información específica que debe divulgarse y la integridad, control y rendición de cuentas (p. 3).

Tipos de reportes que se elaboran:

- Presupuesto. El documento presupuestal es el fundamento de todo el proceso fiscal de un país, su construcción debe permitir señalar el conjunto de ingresos y gastos con suficiente nivel de detalle, como para permitir una evaluación adecuada de las opciones de política y de los costos y beneficios asociados a cada una de ellas. De acuerdo con la OCDE (2010), el presupuesto debe incluir, cuando ello se requiera, información no financiera que permita evaluar el desempeño de los programas de gasto público y estar en contexto con una perspectiva de mediano plazo (dos años sugiere la OCDE, en Colombia ese marco fiscal es de cuatro años).

- Reporte preliminar. Este tipo de documento permite comprender los impactos potenciales del presupuesto y antecede la presentación del presupuesto preliminar (anteproyecto de presupuesto para el caso de Colombia), también debe señalar los objetivos de largo plazo de la política de gasto público.

- Reportes mensuales. Estos se presentan con el fin de analizar, controlar y evaluar el desarrollo de la ejecución presupuestal, así como revisarla en contraste con las metas y objetivos generales de ejecución del presupuesto anual.

- Reportes de medio año. En general este reporte contiene información sobre las ejecuciones fiscales y su relación con las metas presupuestales tanto en términos financieros como no financieros, además, debe incluir información sobre partidas como activos y pasivos no financieros y pasivos contingentes.

- La cuenta pública. Este es el documento final del reporte que recoge todo el resultado de la ejecución presupuestal y los sistemas anexos de esta, es en este sentido, es el principal documento de rendición de cuentas de la gestión fiscal. También, debe analizar el cumplimiento de la metas de gobierno y ser presentado en términos de clasificación del gasto, usando categorías similares de análisis a las empleadas durante la elaboración y definición del documento presupuestal. 
Información específica que se reporta:

- Supuestos y previsiones económicas. La programación presupuestal y su ejecución se escenifican en escenarios económicos que alteran las capacidades del Estado para recaudar impuestos y, por ende, para asignar gasto público, por ello, se hace necesario que dichos supuestos se hagan públicos y se incluyan análisis de sensibilidad de variables claves que afecten el desempeño fiscal.

- Créditos fiscales. Costos estimados que están implícitos en los tratamientos preferenciales que se otorgan en la estructura tributaria. Mientras las reglas de la OECD establecen que deben integrarse al presupuesto los créditos fiscales más importantes, otros esquemas no tienen exigencias específicas sobre el tema o se remiten a las diseñadas por el OECD. Adicionalmente, hay que señalar que las indicaciones de la OECD proponen además un análisis de los créditos fiscales por área y función específica, con el objetivo de proveer información sobre potenciales alternativas fiscales (p. ej. reemplazar el crédito por un programa de gasto público).

Hay que indicar que en ninguno de los modelos de transparencia analizados se proponen términos detallados de la información que deben contener los análisis.

- Activos y pasivos financieros.

- Activos no financieros.

- Obligaciones correspondientes a carga pensional del gobierno.

- Pasivos contingentes.
Integridad de la información, control y rendición de cuentas:

- Políticas contables.

- Sistemas y responsabilidad.

- Auditoría.

- Vigilancia del legislativo y del público.

El cumplimiento de estos criterios permite el desarrollo de procesos de transparencia fiscal, pero adicionalmente hay que tener en cuenta que aspectos como: medios usados para la publicación de información fiscal (página web, medios de comunicación masivos, cartillas o publicaciones), los lenguajes asociados a la divulgación de información fiscal (si el presupuesto se presenta en un lenguaje técnico o en uno accesible a cualquier ciudadano o, al menos, incluye un formato de explicación y presentación) también constituyen factores que permiten evaluar la transparencia de un sistema presupuestal. De tal forma que la transparencia debe garantizar el manejo y compresión presupuestal a partir del acceso abierto a la información.

El Gobierno nacional a través del Ministerio de Hacienda y Crédito Público dio apertura en el 2016 al "Portal de la Transparencia Económica" (MINHACIENDA, 2017), con el fin de acercar a la ciudadanía a la ejecución presupuestal institucional del nivel nacional, con el propósito de fomentar la democratización de la información y reducir los índices de corrupción y promover una cultura de fiscalización y control de los recursos públicos. No obstante, la información suministrada define el porcentaje de ejecución presupuestal por entidad y rubro en el orden nacional, 
no reflejando las ejecuciones presupuestales de los departamentos de Colombia ni la correspondencia con índices de impacto social en criterios de equidad e igualdad.

En el 2003 un equipo de investigadores de Colombia junto con la International Budget Partnership (IBP) aplicaron la encuesta: "Índice Latinoamericano de Transparencia presupuestaria Colombia 2003". El objetivo consistía en analizar horizontalmente la dinámica interna del país junto con los países participantes en el estudio: México, Salvador, Nicaragua, Costa Rica, Ecuador, Perú, Brasil, Chile y Argentina. En este orden de ideas, los resultados de la investigación aportarían valiosa información sobre el grado de participación de los actores sociales externos al gobierno en el tratamiento de la información de los recursos públicos, reflejando así los criterios de equidad, igualdad y justicia como prioridades sociales dentro del esquema de distribución y aplicación del presupuesto, en el Índice de Transparencia Presupuestal (IBP I. B., 2003, p. 4).

Para ello, el estudio se concentró en el análisis de las finanzas del nivel nacional central sobre el grado de percepción de legisladores, organizaciones de la sociedad civil, periodistas e investigadores y representantes de gobernaciones, especialmente de la capital (Bogotá), dejando de lado la información en el orden territorial colombiano, los departamentos, de donde emerge la construcción colectiva del país, especialmente, los municipios capitales como entidad fundamental de la división políticoadministrativa del Estado, escenario en el que se manifiestan las realidades de las políticas sectoriales mediante las ejecuciones presupuestales.

Dentro de los productos de la investigación del Índice Latinoamericano de Transparencia Presupuestaria 2003 se tienen los siguientes:

Tabla 1.

Convocatoria y respuesta de la Encuesta de Percepción del Índice de Transparencia Presupuestal (2003)

\begin{tabular}{|l|c|c|}
\hline Población & Convocada & Total respuestas \\
\hline Legisladores & 44 & 20 \\
\hline Organizaciones Sociedad Civil (OSC) & 36 & 23 \\
\hline Periodistas - columnistas & 23 & 7 \\
\hline Investigadores - académicos & 30 & 17 \\
\hline Total & 133 & 67 \\
\hline$\%$ diputados en población & & 33 \\
\hline$\%$ diputados en respuestas & & 30 \\
\hline$\%$ Coberturas o tasa de respuestas total & & 50 \\
\hline
\end{tabular}

Fuente: índice latinoamericano de transparencia presupuestaria (IBP I. B., 2003, p. 25). 
- En Colombia, la encuesta de percepciones muestra el Índice de transparencia presupuestaria de un máximo de cien puntos, en 44,3 , antes de la encuesta y 44,3 después de la encuesta.

- Se requiere desarrollar un plan de información a través del Sistema Nacional de Información del DANE, SINERGIA o una instancia similar, que garantice mayor fluidez de los datos

- Se debe simplificar y fortalecer los mecanismos de control interno y externo dentro de la administración pública, incluso de manera proactiva proporcionando capacitación e información amplia a la comunidad, ONG, entre otras, para contribuir a mejorar la eficiencia del gasto público.

- La variable participación ciudadana en el presupuesto fue la segunda peor evaluada, con $15 \%$ de respuestas positivas. Esta evaluación muestra la falta de transparencia en su forma más fina, si el presupuesto "no es un problema de cifras y equilibrios, sino una visión de país y de sociedad" (IBP I. B., 2003, pp. 30-36).

Ahora bien, las brechas temporales sobre estudios independientes de transparencia del presupuesto público en Colombia son notorias, lo que imposibilita un seguimiento y control continuo y comparado de los resultados anuales. Además, conviene puntualizar que las investigaciones desarrolladas miden la participación del público a partir de las principales instituciones del poder público del Gobierno nacional y el poder legislativo en un contexto regional latinoamericano y no en el orden de las percepciones territoriales institucionales de los departamentos, lo que subyace un vacío de la disponibilidad de la información en los impactos reales del manejo de los presupuestos públicos en Colombia (publicitación y disponibilidad de la información para la transparencia).

En este orden de ideas, para el 2015, la International Budget Partnership (IBP) mediante la Encuesta de Presupuesto Abierto como "única medida independiente, comparable a nivel mundial, de transparencia presupuestaria, participación y supervisión" (IBP I. B., 2017), aplicó el instrumento en 102 países con el fin de determinar el grado de "acceso a información presupuestaria y oportunidades para participar en todo el proceso presupuestario, [así como la], supervisión por las legislaturas y las instituciones de auditoría [para la contribución] .... un uso más responsable del dinero público" (IBP I. B., 2017). Los resultados de la encuesta regional abierta para Colombia (2015) fueron las siguientes (IBP, 2017):

\section{Mejorar la transparencia}

Colombia debe priorizar las siguientes acciones para mejorar la transparencia presupuestaria:

- Producir y publicar una revisión de mitad de año.

- Aumentar la exhaustividad de la propuesta de presupuesto del Ejecutivo presentando más información sobre la clasificación de los gastos para los años futuros y sobre la clasificación de los ingresos para los años futuros. 
- Aumentar la exhaustividad del informe de fin de año presentando más información sobre los gastos planificados frente a los reales y sobre los ingresos planificados frente a los reales.

\section{Mejorar la participación}

Colombia debe priorizar las siguientes acciones para mejorar la participación presupuestaria:

- Establecer mecanismos creíbles y eficaces (es decir, audiencias públicas, encuestas, grupos focales) para captar una serie de perspectivas públicas sobre asuntos presupuestarios.

- Abrir las audiencias de los informes de auditoría al público para que puedan testificar.

- Publicar informes sobre audiencias del presupuesto público.

\section{Mejorar de la supervisión}

Colombia debe priorizar la siguiente acción para fortalecer la supervisión presupuestaria:

- Establecer consultas regulares sobre asuntos presupuestarios entre el ejecutivo y la legislatura.

\section{RESULTADOS Y DISCUSIÓN}

En otra sección de este documento se había identificado que los niveles territoriales, especialmente en los departamentos, gozan de bajos niveles de participación de la ciudadanía, en la construcción, seguimiento y vigilancia del presupuesto público, este fenómeno es particularmente fuerte en las regiones menos desarrolladas del país, donde las instituciones democráticas aún se muestran débiles e incipientes, y en las que el Estado aún tiene un enorme déficit de presencia.

El conflicto armado se presenta, entonces, como una variable que moldea la capacidad de la sociedad civil para evaluar e incidir en el presupuesto, de hecho, los presupuestos locales y departamentales suelen estar capturados por algún tipo de influencia violenta, tema en el cual no avanzaremos, pero que es un elemento del contexto que no se puede pasar por alto.

Por otra parte, en los niveles departamentales, trabajos anteriores (Gómez y Montesinos, 2014) han identificado bajos niveles de calidad y difusión de la información presupuestal y fiscal, la cual también se presenta en un contexto de uso ineficiente de los recursos públi$\cos$, que limita la capacidad para atender las necesidades de desarrollo social y, particularmente, aquellas que se relacionan de manera más estrecha con los elementos del posconflicto/posacuerdo.

A pesar de la implementación de herramientas como el e-goverment, la debilidad de la capacidad institucional de los departamentos es evidente: los reportes de información fiscal y financiera son informales o inexistentes, están sujetos a la voluntad del corpus administrativo local o simplemente no representan un punto de interés para el desarrollo de rutinas administrativas.

Sin embargo, las aproximaciones para evaluar los niveles de transparencia 
no han sido consistentes y se han concentrado en evaluar (como en el ya citado trabajo de Gómez y Montesinos, 2014) la difusión de la información por medio de herramientas de gobierno electrónico, sin analizar otros aspectos fundamentales de la transparencia, ya descritos en la primera parte de este documento.

\section{El Índice de Transparencia Presupuestal}

En este sentido, la construcción del Índice de Transparencia Presupuestal de los departamentos es una herramienta que permite evaluar las condiciones de divulgación, uso y comprensión de la información presupuestal elaborada por los niveles centrales de los gobiernos departamentales en Colombia, así como los escenarios que a partir de esta información le permiten a los entes de control y a la ciudadanía incidir en los procesos de planeación, formulación, ejecución y evaluación y control del presupuesto público.

El índice sintetiza y sistematiza los desempeños de los fiscos departamentales en cuatro aspectos relevantes:

\section{a. Disponibilidad de la información} presupuestal. Esto implica evaluar si las autoridades fiscales departamentales publican los documentos más importantes que componen cada una de las fases del presupuesto público y si lo hacen de manera periódica de acuerdo con la estructura legal del ciclo presupuestal. Incluye, además, una revisión de los niveles de accesibilidad o facilidad para acceder a la información divulgada. b. Oportunidad de la información. Cada una de las etapas del ciclo presupuestal requiere de un proceso de producción de información previo, para que los diferentes interesados (asambleas, entes de control, ciudadanos) puedan tomar decisiones e incidir en la propuesta presupuestal elaborada. La publicidad de la información debe ser oportuna y no producirse con posterioridad a la decisión pertinente.

\section{c. Comprensibilidad de la información.}

El presupuesto público es un documento con un alto nivel de complejidad técnica, en este sentido, es un saber especializado que no necesariamente es comprensible por parte de todos los actores que concurren en el debate presupuestal. Esta dimensión permite evaluar la forma en que las autoridades fiscales producen información presupuestal en niveles de lenguaje que permitan una mejor comprensión de esta a todos los ciudadanos.

\section{d. Integralidad de la información. Esto} implica, por una parte, la presentación de información presupuestal relevante a todos los rubros del presupuesto, y, por otra, la presentación de información para todas y cada una de las fases del ciclo presupuestal.

Para evaluar estos aspectos, se aplicarán tres metodologías de medición complementaria:

1. Una revisión de la información presupuestal disponible en las plataformas electrónicas oficiales de las gobernaciones y contralorías departamentales. 
2. El diligenciamiento de una encuesta electrónica dirigida a los funcionarios encargados de la gestión presupuestal en el nivel central de los departamentos.

3. La aplicación de una entrevista en profundidad a funcionarios de alto nivel encargados de la gestión presupuestal. Esta entrevista se realizará solamente a algunas de las entidades departamentales y servirá para complementar información identificada en los otros pasos.
Al final de este proceso, se sintetizará y consolidará la información recaudada y se procederá a la elaboración del índice y el ranking de entidades departamentales, junto con un análisis comparativo por tipologías de departamentos y una serie de recomendaciones, las cuales serán socializadas con las autoridades fiscales por medio de un documento ejecutivo, así como en un evento en el que se presentarán los resultados del ejercicio (ver gráfico 1).

\section{Gráfico 1.}

Etapas en la construcción del Índice de Transparencia Presupuestal 2018
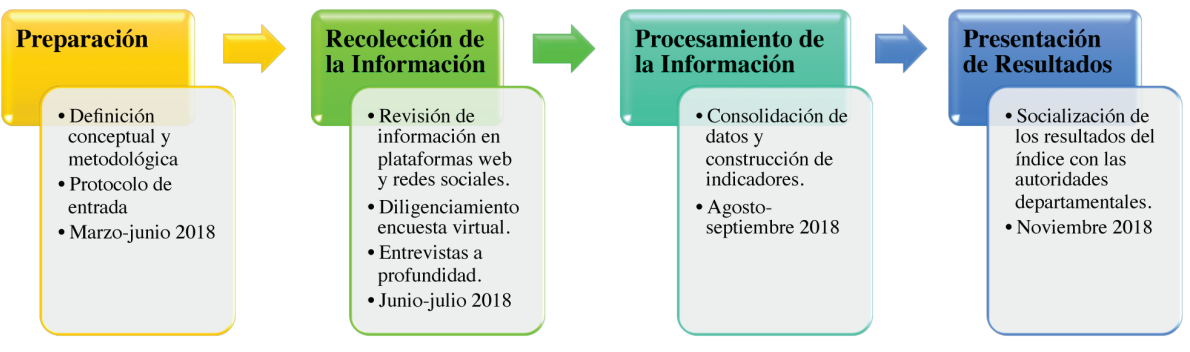

Fuente: elaboración propia.

\section{Resultados esperados}

La construcción del Índice de Transparencia Presupuestal busca:

- Proveer a las autoridades fiscales de los departamentos herramientas para evaluar los procesos de producción y visibilización de la información presupuestal.

- Apoyar a las autoridades departamentales en los procesos de mejora de sus niveles de revelación de información presupuestal.

- Aportar a la construcción de una ciudadanía con mayores niveles de conocimiento de los asuntos presupuestales y con capacidad de comprender y legitimar el uso de los recursos públicos.

- Generar procesos sinérgicos de cooperación entre la ESAP y las autoridades departamentales para el fortalecimiento de la gestión pública, la construcción de escenarios de buen gobierno y la mejora de los niveles de transparencia en la gestión de las entidades territoriales. 


\section{CONCLUSIONES}

La transparencia presupuestal se ha convertido en una exigencia cada vez más poderosa en la gestión de los recursos presupuestales, basado en la idea de que la ciudadanía es la "dueña" de los recursos públicos, una democracia no puede funcionar si las cuentas públicas aparecen opacas para sus ciudadanos.

Esta idea ha trascendido al punto de que instituciones multilaterales, organizaciones de países (como OCDE) y esfuerzos de la sociedad civil a nivel global y regional, se hayan esforzado por promover el desarrollo de criterios y procedimientos de transparencia de la información fiscal y financiera de las entidades públicas.

En Colombia, el nivel central de gobierno ha desarrollado procedimientos y plataformas importantes que han aumentado el nivel de transparencia del presupuesto, sin embargo, el camino aún es largo, pues el país se sitúa en las evaluaciones internacionales en lugares más bien bajos en la calidad y amplitud de sus prácticas de TP.

Esta situación es aún más preocupante en los niveles subnacionales, en los que la capacidad institucional es muy baja, la presencia del conflicto armado hace mella en la capacidad de las administraciones para el desarrollo de unos procedimientos adecuados de transparencia y donde la participación ciudadana es muy débil.

La medición y evaluación de la tp en los niveles subnacionales, especialmente en los departamentos, supone un paso en el sentido de identificar criterios estándar en la producción y revelación de información, medirlos en cada departamento y producir recomendaciones de prácticas de transparencia, posicionando el tema en las agendas de los gobiernos departamentales.

\section{REFERENCIAS BIBLIOGRÁFICAS}

Álvarez, D. A. (2006). Transparencia y rendición de cuentas en los presupuestos de defensa: del sector al escrutinio público. Boletín del programa seguridad y ciudadanía $\mathrm{n}^{\circ} 1$.

Bastida, F. y Benito, B. (2007). Central Government Budget Practices and Transparency: an International Comparison. Public Administration, 85, Issue 3, 667-716.

Bautista, J. A. y Sepúlveda D. A. (2016). Transparencia del gasto fiscal en Colombia. Science of Human Action, 1(2).

Beghin, N. y Zigoni, C. (2014). Avaliando os websites de transparência orçamentária nacionais e sub- nacionais e medindo impactos de dados abertos sobre direitos humanos no Brasil/ Zigoni. Brasilia: Instituto de Estudos Socioeconômicos.

Bessire, D. (2005). Transparency: a twoway mirror? International Journal of Social Economics, 32(5), 424-438.

Betriu, M. L. (2003). La transparencia presupuestaria. Problemas y soluciones. Glennerster, R. y Shin, Y. (2003). Is Transparency Good for You, and Can the IMF Help? Working Paper 03/132. Washington D. C.: International Monetary Fund. 
BID, B. I. (2006). La economía del proceso presupuestal en Colombia. Bogotá: BID.

FMI, F. M. (2012). Transparencia fiscal, rendición de cuentas y riesgo fiscal. FMI.

Gómez Villegas, M. y Montesinos J. V. (2014). Gobierno electrónico y transparencia de la información financiera y presupuestal de los departamentos en Colombia.Revista Venezolana de Gerencia, 19(68), 670-698.

Heald, D. (2003). Fiscal Transparency: Concepts, Measurement and UK Practice.

International Budget Partnership. (10 de marzo del 2015). IPB. Recuperado de http://internationalbudget.org

IBP, I. B. (2003). Índice Latinoamericano de Transparencia Presupuestaria 2003. Una comparación de 10 países. Bogotá: International Budget Partnership.

IBP, I. B . (04 de junio del 2017). International Budget Partnertship. Open Budgets Transform Lives. Recuperado de http://www.internationalbudget. org/opening-budgets/open-budgetinitiative/open-budget-survey/

International Budget Partnership. (10 de marzo del 2015). IPB. Recuperado de http://internationalbudget.org

Minhacienda, M. D. (20 de enero del 2017). Portal de transparencia económica. Recuperado de http://www.pte.gov. co/WebsitePTE/

Moreira Corrêa, I. y Claussen Spinelli, M. V. (2011). Políticas de transparencia en la administración pública brasileña. Revista del CLAD Reforma y Democracia, (51), 129-152.
OECD. (2010). OCDE Mejores prácticas para la transparencia presupuestaria. OCDE. Recuperado de http:// internationalbudget.org/wp-content/ uploads/OECDBestPracticesSpanish.pdf

Paprocki, L. M. (2017). Analisis de las variables para la valoración de la transparencia en la administración de municipios. "Visión de Futuro", 21(1), 1-2.

Platt Neto, O. A., Cruz, F., Rolim Ensslin, S. y Ensslin, L. (2007). Publicidade e Transparência das Contas Públicas: obrigatoriedade e abrangência desses principios na administração pública brasileira. Contabilidade Vista y Revista, 18(1), 75-94.

Pires, V. (2014). Transparencia presupuestaria en los gobiernos municipales de Brasil por medio de internet: el caso de Piracicaba, São Paulo. Poder Local, 19, 46-54.

Poterba, J. M. y Von Hagen, J. (1999). Introduction. En J. M. Poterba y J. Von Hagen (eds.), Fiscal Institutions and Fiscal Performance (pp. 1-12). Chicago: University of Chicago Press.

Rausch, R. B. y Maurélio, S. (2010). Controle social na administração pública: a importância da transparência das contas públicas para inibir a corrupção.

Renzio, P. y Angemi, D. (2011). Comrades or culprits? Comrades or culprits? Comrades or culprits? in Aid Dependent Countries. Ibei Working Papers, 33, 3-24.

Ríos, A. M. y Benito, B. (2016). Factors Explaining Public Participation in the Central Government Budget Process. Australian Journal of Public Administration, 76(1), 48-64. 
Ríos, A. M., Guillamon, M. D., Benito, B. y Bastida, F. (2017). The influence of transparency on budget forecast deviations. Journal of Forecasting Department of Accounting and Finance, 1-18. 